\title{
Iniciativas de Governança de TIC que sugerem Modelos, Técnicas e Boas Práticas para a Administração Pública Federal: um Mapeamento Sistemático
}

\author{
Marianne B. Diniz da Silva ${ }^{1}$, Isabel D. Nunes ${ }^{2}$, Rogerio P. C. do Nascimento ${ }^{1}$, \\ Maria Augusta S. N. Nunes ${ }^{1}$ \\ ${ }^{1}$ Programa de Pós-Graduação em Ciência da Computação (PROCC) Universidade \\ Federal de Sergipe (UFS) - São Cristóvão, SE - Brasil \\ ${ }^{2}$ Instituto Metrópole Digital (IMD - UFRN), Natal - RN - Brasil. \\ \{mariannedinizsi, rogerio.33, gutanunes\}@gmail.com, bel@imd.ufrn.br
}

\begin{abstract}
ICT Governance in Public Administration is a composition of ICT processes and initiatives with the aim of aligning IT with the business that collaborates for the best performance of the organization. The adoption of ICT Governance in institutions is still considered a challenge. Therefore, this article aims to present a systematic mapping in order to identify and systematize in which countries there are initiatives for ICT Governance in the Federal Public Administration (APF). Thus, this article reports which methods and / or techniques are used in the APF and highlights which Universities and Authors have contributed in this field.
\end{abstract}

Resumo. Governança de TIC na Administração Pública é uma composição de processos e iniciativas de TIC com o intuito de alinhamento de TI com o negócio que colabora para o melhor desempenho da organização. A Adoção de Governança de TIC nas instituições ainda é considerada um desafio. Portanto, este artigo objetiva apresentar um mapeamento sistemático a fim de identificar e sistematizar em quais países existe iniciativas para Governança de TIC na Administração Pública Federal (APF). Assim, este artigo relata quais métodos elou técnicas são utilizados na APF e destaca quais Universidades e Autores têm contribuído neste campo.

\section{Introdução}

Segundo o Information Technology Governance Institute - ITGI (2016) a Governança de TIC é uma estrutura de relações e processos que monitora e controla uma organização com o intuito de promover valor ao negócio por meio do equilíbrio entre risco e retorno dos investimentos em TI. Governança de TIC tem sido utilizada de forma crescente como um modelo de estrutura para esta tarefa de gestão por meio dos seus frameworks apoiados em boas práticas do mercado e aderentes às regulamentações externas.

A Governança de TIC e o alinhamento da gestão da TIC caminham junto com o planejamento estratégico como forma de agregar valor à utilização dos produtos e serviços de TIC por meio do investimento ideal para obtenção de vantagens competitivas 
e retorno financeiro, conceitos estes mais relacionados, na maioria dos casos, às empresas privadas e não aos órgãos do setor público [Carvalho 2006].

Por mais que haja um grande reconhecimento sobre a importância da Governança de TIC e o seu papel nas organizações, a forma de como implementá-la na prática ainda é um grande desafio e depende de cada caso. A aplicação em órgãos públicos torna o caso ainda mais específico e complexo. Com isso faz-se necessário então investigar a implantação da Governança de TIC no ambiente real, acompanhando o processo de perto para identificar problemas reais, planejar soluções, introduzir mudanças e refletir sobre os resultados obtidos [Mauro and Augusto 2010].

Este mapeamento sistemático tem como objetivo identificar e sistematizar em quais países existem iniciativas para a Governança de TIC na sua Administração Pública Federal (APF) e quais métodos e/ou técnicas são utilizados. Para isso, foram mapeados artigos em diversas bases de dados na área de Computação.

Este artigo encontra-se organizado da seguinte forma: na seção 2 apresenta-se o método adotado nesse mapeamento. Na seção 3, descreve-se a análise dos resultados e na seção 4 apresentam-se as considerações finais e alguns possíveis trabalhos futuros.

\section{Método}

Com o objetivo de estudar e mapear em quais países existe iniciativas para a Governança de TIC na APF e quais os métodos e/ou técnicas são utilizados, foi escolhido o método de mapeamento sistemático (SLM, Systematic Literature Mapping). Para Petersen et al. (2008), a SLM é definida como um estudo secundário, pois analisa estudos primários com o objetivo de integrar e/ou sintetizar evidências. Esse estudo possui como finalidade prover uma visão geral de uma determinada área, questão de pesquisa e/ou fenômeno, identificando e analisando os tipos de pesquisa relevantes e disponíveis [KITCHENHAM 2004].

Nesta seção é descrito os procedimentos metodológicos utilizados para conduzir este estudo. Para isso, faz-se necessário definir as questões de pesquisas, a estratégia de busca e os critérios de seleção dos artigos.

Nas subseções que seguem têm-se o detalhamento do processo de busca e as seleções dos estudos primários. Desta forma, torna-se possível a avaliação e repetição da revisão por outros pesquisadores.

\subsection{Questões de Pesquisa}

O escopo para a aplicação deste mapeamento sistemática relaciona-se em identificar o estado de Governança de TIC na Administração Pública. Dessa forma, foram elaboradas quatro questões de pesquisa para atingir-se o objetivo deste mapeamento:

Q1. Em quais países existe iniciativas para a Governança de TIC na Administração Pública Federal (APF)?

Q2. Quais métodos e/ou técnicas de Governança de TIC são sugeridos para a Administração Pública Federal (APF)?

Q3. Quais as universidades que mais contribuem para a literatura da área?

Q4. Quem são os autores mais produtivos nesta área?

SILVA, M. B. D.; NUNES, I. D.; NASCIMENTO, R. P. C; NUNES, M. A. S. N.

Iniciativas de Governança de TIC que sugerem Modelos, Técnicas e Boas Práticas para a Administração Pública Federal: um Mapeamento Sistemático

isys | Revista Brasileira de Sistemas de Informação, Rio de Janeiro, vol. 10, No. 1, pp. 39-51, 2017 


\subsection{Estratégia de Busca e de Seleção}

As bases de dados na área de computação selecionadas para a execução da busca foram: Scopus e a Biblioteca Digital Brasileira de Computação (BDBComp). O portal de periódicos da $\mathrm{CAPES}^{1}$ foram utilizados para o download dos artigos sem restrições nas duas bases.

Para a execução da busca na base da Scopus foram utilizadas ferramentas de filtragem avançada considerando o resumo (abstract) dos artigos, os idiomas (português e inglês) e a área de pesquisa (Ciência da Computação) com o intuito de minimizar a quantidade dos artigos que não contemplam o escopo de Governança de TIC na Administração Pública Federal (APF).

Já para a base BDBComp, foi realizada uma pesquisa básica, visto que a base não possui mecanismos avançados para a busca.

As palavras chaves definidas para a busca foram:

- Em inglês: Information, Communication, Technology, Governance, Management, Public, Administration.

- Em português: Tecnologia, Informação, Comunicação, Governança, Gestão, Administração, Pública, Federal.

As strings de busca utilizadas para as bases ficaram definidas como:

- Scopus: ABS(((“Information communication technology") OR (“ICT”) OR ("IT") AND ("governance" OR "management" OR "strategic planning") AND ("public administration"))) AND (LIMIT-TO(SUBJAREA, "COMP”), AND (LIMIT-TO(LINGUAGE), "English") OR (LIMIT-TO, "Potuguese") )

- BDBComp: Como não existe meios de busca avançada, foram utilizadas para as buscas as palavras chaves tanto em inglês como português, por meio de buscas isoladas para cada termo (("Governança TI" OR "Governança TIC" OR "Administração Pública Federal”) OR ("Governance IT" OR "Governance ITC" OR "Public, Administration"))

As pesquisas com as strings de busca foram realizadas no mês de maio de 2016. Para a busca na base da Scopus foram utilizadas apenas as palavras chaves em inglês retornados 395 artigos. Já para a busca na Biblioteca Digital Brasileira de Computação (BDBComp) foram utilizados para a busca palavras chaves em português e inglês, no qual foram encontrados 2 artigos. Totalizando 397 artigos encontrados.

Não foi encontrado nenhum artigo que possuía as palavras chaves em inglês na base BDBComp,

Percebe-se que o maior número de artigos está na base da Scopus, visto que ela contempla artigos de diversas bases (ACM, IEEE, ScienceDirect e outras). Essas bases que compõe a Scopus são frequentemente utilizadas por pesquisadores de Ciência da Computação [SCOPUS 2016].

Finalizado as buscas, iniciou-se a seleção dos artigos encontrados com base nos critérios e procedimentos de seleção.

\footnotetext{
${ }^{1}$ https://www.periodicos.capes.gov.br/

SILVA, M. B. D.; NUNES, I. D.; NASCIMENTO, R. P. C; NUNES, M. A. S. N.

Iniciativas de Governança de TIC que sugerem Modelos, Técnicas e Boas Práticas para a Administração Pública Federal: um Mapeamento Sistemático

isys | Revista Brasileira de Sistemas de Informação, Rio de Janeiro, vol. 10, No. 1, pp. 39-51, 2017
} 


\subsection{Critérios e procedimentos de seleção de estudos}

Com o intuito de selecionar os artigos relevantes para o objetivo deste mapeamento foram definidos critérios de inclusão e exclusão dos artigos. Para inclusão foram definidos os seguintes critérios:

1)Foram incluídos artigos com foco em Governança de TIC na Administração Pública;

2)Foram incluídos artigos que apresentaram descrição detalhada de modelos, técnicas, tecnologias e boas práticas utilizadas na Governança de TIC na Administração Pública Federal;

3)Foram incluídos artigos que possuía ano de publicação superior a 2006;

4)Foram incluídos artigos no qual as suas fontes fossem conferências ou Journals, ou seja, artigos de conferências ou journals.

O critério de inclusão dos artigos foi por meio da análise do resumo (abstract) dos artigos e conclusão (conclusion) de cada artigo encontrado.

Já para exclusão foram definidos os seguintes critérios:

1)Foram excluídos artigos duplicados.

2)Foram excluídos artigos que não estavam disponíveis para visualização do texto completo.

3)Foram excluídos artigos que foram publicados com data inferior a 2006.

4)Foram excluídos artigos que não descrevesse métodos e/ou técnicas para a Governança de TIC na APF.

Com a adoção dos critérios de inclusão e exclusão dos artigos, foram realizadas as avaliações para responder as questões. Do total de 397 artigos encontrados, 56 foram selecionados para compor as respostas do mapeamento, sendo que um artigo foi encontrado duplicado.

A Figura 1 mostra o processo de busca e seleção dos artigos realizados neste mapeamento. Durante a execução do processo de busca e seleção dos artigos, foram realizadas análises detalhadas, com o propósito de identificar os artigos que mais se enquadrassem com o objetivo proposto.

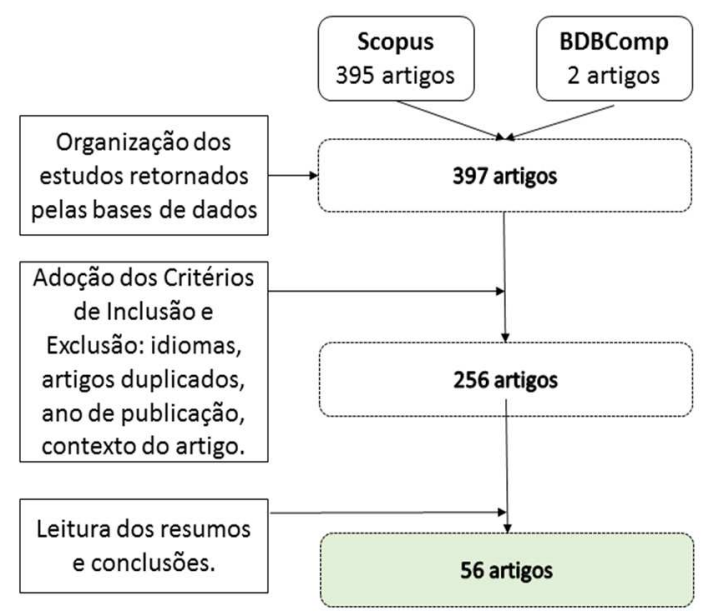

Figura 1. Processo de busca e adoção dos critérios de inclusão e exclusão.

SILVA, M. B. D.; NUNES, I. D.; NASCIMENTO, R. P. C; NUNES, M. A. S. N.

Iniciativas de Governança de TIC que sugerem Modelos, Técnicas e Boas Práticas para a Administração Pública Federal: um Mapeamento Sistemático

isys | Revista Brasileira de Sistemas de Informação, Rio de Janeiro, vol. 10, No. 1, pp. 39-51, 2017 
Com a aplicação da String de busca e palavras chaves nas bases foram encontrados 397 artigos na busca, após a adoção dos critérios de inclusão e exclusão obteve-se 256 artigos. Com os 256 artigos realizou-se a leitura dos resumos e conclusão, houve uma grande redução do número de artigos. Essa redução se deu por meio dos critérios de exclusão focando no contexto do artigo, no qual foram selecionados apenas 54 artigos na Base da Scopus e na BDBComp a seleção foi dos 2 artigos. A identificação completa dos estudos primários pode ser encontrada na seção de Referências deste artigo.

Após a realização da seleção, os estudos primários foram encaminhados para a leitura do resumo e análise, os resultados dessa etapa podem ser encontrados na seção 3.

\section{Análise dos Resultados}

Nesta seção, apresentam-se os resultados da análise dos estudos primários, respondendo às questões de pesquisa apresentadas na seção 2 deste mapeamento.

A resposta para a questão de pesquisa Q1, Em quais países existe iniciativas para a Governança de TIC na Administração Pública Federal (APF)?, é ilustrada na Tabela 1, a qual mostra países que possuem alguma preocupação com a Governança de TIC para Administração Pública Federal (APF).

Tabela 1. Países que possuem alguma iniciativa para a Governança de TIC na APF.

\begin{tabular}{|c|c|}
\hline Países & Referências \\
\hline Afeganistão & [Mohmand et al. 2010] \\
\hline Alemanha & [Becker et al. 2009] \\
\hline Argentina & [Falivene and Silva 2008] \\
\hline Brasil & [Mauro and Augusto 2010] \\
\hline China & [Lin and Fong 2013] \\
\hline Colômbia & [Rodríguez 2013] \\
\hline Croácia & [Šimundić and Boban 2009] \\
\hline Eslovênia & [Decman and Klun 2008][Dobnikar and Nemec 2007] \\
\hline Espanha & $\begin{array}{l}\text { [Cerrillo-i-Martínez 2012][De Juana-Espinosa et al. 2012][Gonzalez et al. } \\
\text { 2007] }\end{array}$ \\
\hline Finlândia & [Valtonen and Leppänen 2009][Liimatainen and Seppänen 2008] \\
\hline Hungria & [Laszlo and Szuts 2006] \\
\hline Índia & $\begin{array}{l}\text { [Patra and Das 2014][Dwivedi and Bharti 2010][Verma and Mishra } \\
\text { 2009][Pardhasaradhi and Ahmed 2007] }\end{array}$ \\
\hline Irlanda & [Bannister 2006] \\
\hline Itália & $\begin{array}{l}\text { [Consoli et al. 2015][Casagranda et al. 2014][Reggi et al. 2014][Suppa et } \\
\text { al. 2013][Casalino and Draoli 2010][Cabiddu 2010][Bigliardi and Dormio } \\
\text { 2009][Castelnovo 2009] }\end{array}$ \\
\hline Japão & [Nguyen 2008] \\
\hline Macedônia & [Bogoevska and Plastinovski 2010] \\
\hline Madagascar & [Rakotonirina and Raoelson 2013] \\
\hline Malásia & [Azmi et al. 2015] \\
\hline
\end{tabular}

SILVA, M. B. D.; NUNES, I. D.; NASCIMENTO, R. P. C; NUNES, M. A. S. N.

Iniciativas de Governança de TIC que sugerem Modelos, Técnicas e Boas Práticas para a Administração Pública Federal: um Mapeamento Sistemático

isys | Revista Brasileira de Sistemas de Informação, Rio de Janeiro, vol. 10, No. 1, pp. 39-51, 2017 


\begin{tabular}{l|l}
\hline Mongólia & {$[$ Dandar et al. 2007] } \\
\hline Nigéria & {$[$ Adeyeye and Aladesanmi 2011] } \\
\hline Nova Zelândia & {$[$ Lips 2012] } \\
\hline Paraguai & {$[$ Cernuzzi and Pane 2014] } \\
\hline Portugal & {$[$ Esteves and Alves 2013][Santos et al. 2013] } \\
\hline Quênia & {$[$ Bernardi 2009] } \\
\hline República Checa & [Capek and Ritschelova 2010] \\
\hline República Dominicana & [Galva 2011] \\
\hline Romênia & {$[$ Mătuescu and Glăvan 2012] } \\
\hline Suécia & [Juell-Skielse and Perjons 2009] \\
\hline Tailândia & [Jackson and Chongthammakun 2011] \\
\hline Turquia & [Tsohou et al. 2013] \\
\hline Uruguai & [Garbarino et al. 2011] \\
\hline Vietnã & [Nguyen 2008] \\
\hline
\end{tabular}

De acordo com esses resultados, verifica-se que os países com maior quantidade de referências sobre a Governança de TIC na Administração Pública Federal são a Itália, a Índia e a Espanha. Os demais apresentam apenas uma ou duas referências.

A resposta para a questão de pesquisa Q2, Quais métodos e/ou técnicas de Governança de TIC são sugeridos para a Administração Pública Federal (APF)?, é ilustrada na Tabela 2, a qual mostra os métodos e/ou técnicas de Governança de TIC para a Administração Pública Federal (APF).

Tabela 2. Métodos e/ou técnicas de Governança de TIC na APF.

\begin{tabular}{l|l}
\hline \multicolumn{1}{c|}{ Métodos e/ou técnicas } & \multicolumn{1}{c}{ Referências } \\
\hline ADONIS & {$[$ Emmanouil and Sotirios 2009] } \\
\hline BSC & {$[$ Niehaves et al. 2007] } \\
\hline COBIT & {$[$ Mauro and Augusto 2010] } \\
\hline GERTI & [Briganó et al. 2012] \\
\hline ISO/IEC 27001 & [Stoll and Laner 2010a][Stoll and Laner 2010b] \\
\hline ISO/IEC 20000-1 & [Stoll and Laner 2010a] \\
\hline ITIL & [Esteves and Alves 2013] \\
\hline PICTURE & [Becker et al. 2009][Becker et al. 2008][Baacke et al. 2007] \\
\hline PMBOK & [Santos and Varajão 2015][Pankowska 2011] \\
\hline SAKE & [Ko et al. 2011] \\
\hline SISMAP & {$[$ Galva 2011] } \\
\hline DEA & [Tsohou et al. 2013] \\
\hline
\end{tabular}

De acordo com esses resultados verifica-se que os métodos e/ou técnicas com maiores quantidades de referências para aplicação na Governança de TIC com ênfase na 
Administração Pública Federal foram PICTURE, PMBOK e ISO/IEC 27001. Os demais métodos e técnicas apresentaram apenas uma referência.

A reposta para a questão de pesquisa Q3, Quais as universidades que mais contribuem para a literatura da área?, pode ser visualizada na Figura 2. As universidades são: European Research Center for Information Systems, Universitat d'Alacant e Jyvaskylan Yliopisto, ambas com 2 artigos cada.

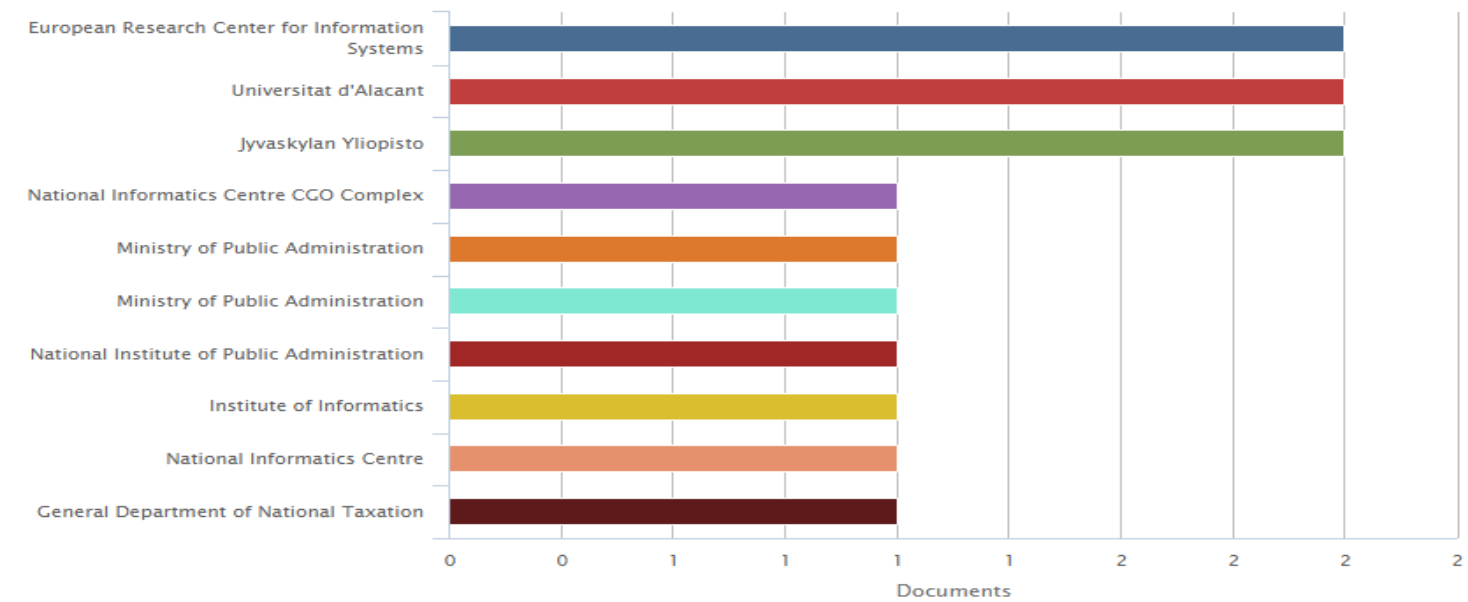

nesta área?, pode ser visualizada na. Figura 3. Os autores enscontrados são: Becker, com 3 artigos, Laner, Bergener, Rackers e Stolt ambos com 2 artigos cada.

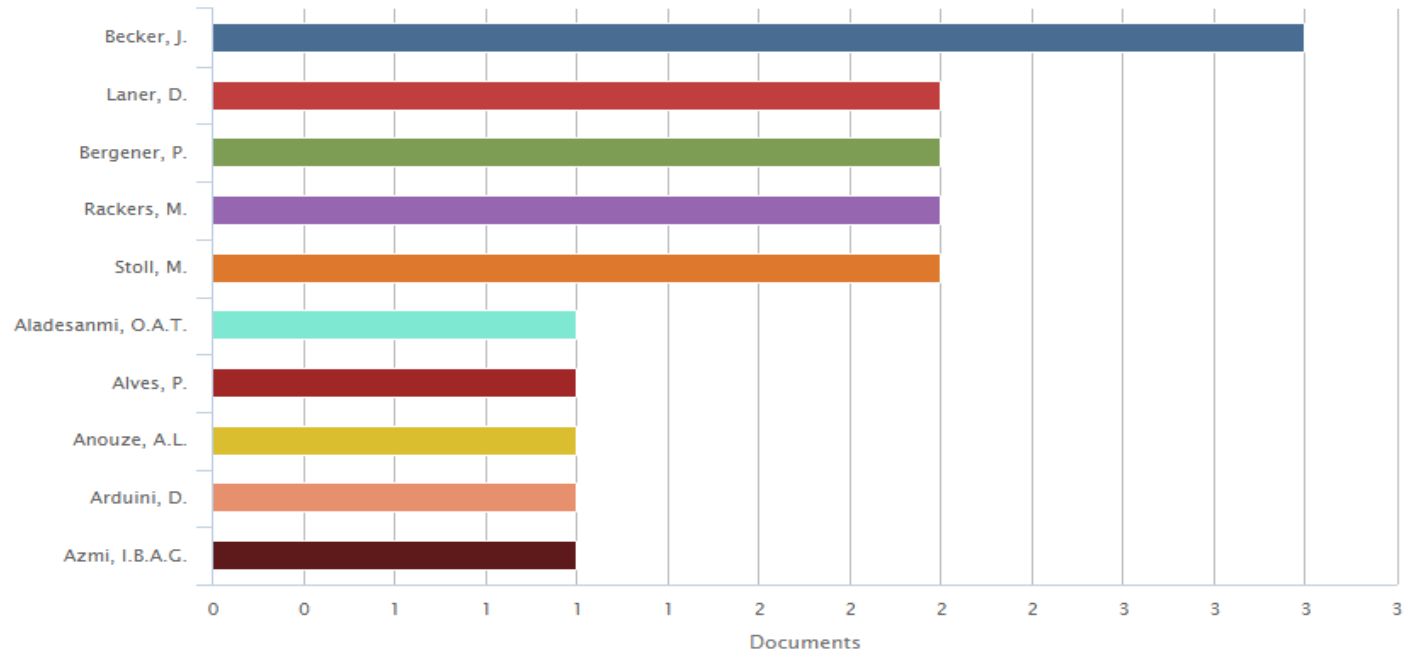

Figura 3. Gráfico dos autores que mais publicam na sobre Governança de TIC na APF.

Apesar do pequeno número de trabalhos selecionados, o mapeamento respondeu satisfatoriamente as perguntas de pesquisas propostas.

A primeira pergunta de pesquisa visava descobrir em quais países existem iniciativas para a Governança de TIC na APF. Respondendo a esta pergunta, o mapeamento identificou 32 países. Já para segunda pergunta que visava verificar os métodos e/ou técnicas de Governança de TIC na APF, o mapeamento retornou 12 técnicas e métodos. E para terceira e quarta pergunta o mapeamento também respondeu como ilustrou as Figuras 2 e 3. 
A pesquisa foi realizada com o objetivo de guiar a autora, sobre como percorre a Governança de TIC na APF nos últimos 10 anos em outros países e quais métodos e técnicas já existentes. Com os resultados mapeados pretende-se detalhar a Governança em cada país e o funcionamento dos métodos e técnicas.

\section{Ameaças à validade}

As ameaças à validade do presente estudo foram:

Viés de seleção: no início do processo, aplicou-se os critérios de inclusão e exclusão com base em nosso julgamento e os estudos foram incluídos ou excluídos neste estudo de mapeamento. Isso significa que alguns estudos podem ter sido categorizados incorretamente. Com a intenção de mitigar esta ameaça, discutimos o protocolo do estudo entre os pesquisadores para garantir o entendimento comum deste. Depois disso, dois pesquisadores analisaram cada artigo e o terceiro e quarto pesquisador quando necessário.

Validade de construção: as questões de pesquisa e as strings de busca definidas neste estudo podem não abranger a área de Ciência da Computação relacionadas às iniciativas de Governança de TIC que sugerem Modelos, Técnicas e Boas Práticas para a APF. Para reduzir esse viés, alguns especialistas em Governança de TIC e Gestores de TIC de Organizações Públicas foram consultados. Depois disso, foi discutido sobre os termos selecionados e, finalmente, definiu as perguntas de pesquisa e strings de busca.

Extração de dados: viés ou problema de extração pode afetar a caracterização das abordagens e análise de estudos selecionados. A fim de reduzir este viés, discutimos profundamente as categorias e as formas de extração. A extração de dados foi realizada conjuntamente por dois pesquisadores. Se os pesquisadores discordarem sobre uma classificação ou informações extraídas, uma terceira opinião foi considerada para resolver as diferenças e para garantir que os dados extraídos sejam válidos e claros para análises posteriores.

Validade externa: realizamos um estudo de mapeamento sistemático sobre os estudos publicados a partir de 2006 nas bases da Scopus e BDBComp. Este ponto implica que podemos ter perdido alguns estudos relevantes contidos nestas bases de dados e em outras bases que não foram utilizadas. Assim, não podemos generalizar nossas conclusões de todas as abordagens para promover iniciativas de Governança de TIC na APF. No entanto, nossos resultados podem apoiar futuras investigações nessa área.

\section{Considerações Finais}

Neste trabalho foi realizado um mapeamento sistemático cujo objetivo foi identificar e analisar quais países se preocupam com a Governança de TIC na APF e quais métodos e/ou técnicas são mais utilizados. Quatro questões foram levantadas para guiar o mapeamento sistemático e após a avaliação de um total de 397 estudos inicialmente retornados, 56 foram selecionados como estudos relevantes para o mapeamento.

A partir da análise dos estudos selecionados foram apresentados mecanismos que auxiliam como guia sobre a Governança de TIC na APF em outros países e quais a técnicas e/ou métodos existentes. Além disto, foram apresentados as Universidades que mais pesquisam nessa área e quais os autores que estão mais publicando, nos quais foram respostas para a questão 3 e 4 . 
Vários países têm demonstrado iniciativas para a Governança de TIC na APF. No entanto, os que tiveram maiores envolvimento com pesquisas foram Itália, Índia e Espanha.

Nesse mapeamento identificou-se também quais métodos e/ou técnicas são utilizados na Governança de TIC na APF. Aqueles que tiveram maior destaque foram ISO/IEC 27001, PICTURE e PMBOK.

Quanto às universidades que mais publicam na área destacaram-se a Europen Research Center for Information Systems, Universitat d'Alacant e Jyvaskylan Ylioposisto, ambas com duas publicações.

Por fim, buscou-se saber quais são os autores que mais publicavam. Dentre eles, destacaram-se Becker, com 3 artigos e Laner, Bergener Rackers e Stoll ambos com dois artigos.

Um ponto a se ressaltar é que em nenhum dos artigos coletados e selecionados foi encontrado relato de utilização do Capability Maturity Model Integration (CMMI) e The Open Group Architecture (TOGAF) entre outros, que são conhecidos como métodos de Governança de TIC na academia e largamente usados na indústria brasileira.

Com isso, acredita-se que esta pesquisa apresenta resultados relevantes à academia, listando quais os países que estão mais preocupados com a Governança de TIC na APF e quais são os métodos e/ou técnicas utilizadas. Neste sentido, este artigo pretende ser uma fonte de consulta relevante para esta área de pesquisa.

Como objetivo futuro, pode-se destacar inicialmente o detalhamento da forma de utilização ou adoção dos métodos e técnicas encontradas com o resultado deste trabalho. Como também a expansão dos estudos para a área de administração, com o intuito de verificar se em administração existe a utilização dos métodos de Governança conhecidos na indústria que são eles: CMMI, TOGAF entre outros.

Outra oportunidade é detalhar (práticas, auditórias, entre outros) como cada um desses países aborda a Governança de TIC na APF.

\section{Referências Bibliográficas}

Adeyeye, M. O. and Aladesanmi, O. A. T. (2011). Re-inventing local government capacity in Nigeria: The e-governance imperative. In MIPRO 2011 - 34th International Convention on Information and Communication Technology, Electronics and Microelectronics - Proceedings.

Azmi, I. B. A. G. ., Ismail, S. H. B. S. ., Basir, S. A. B. ., Norman, A. A. B. . and Yusof, R. J. B. R. . (2015). Application of wasatiyyah concept in public administration performance appraisal system in Malaysia. Advanced Science Letters, v. 21, n. 5, p. $1307-1310$.

Baacke, L., Fitterer, R. and Rohner, P. (2007). Measuring impacts of ICT on the process landscape of public administrations. In ICEG 2007 - 3rd International Conference on e-Government. . Academic Conferences Limited.

Bannister, F. (2006). Government by wire: Decentralization, (R) e-Location and technology. In Proceedings of the European Conference on e-Government, ECEG. .

Becker, J., Bergener, P., Kleist, S., Pfeifer, D. and Räckers, M. (2008). Business process model-based evaluation of ICT investments in public administrations. In 14th Americas Conference on Information Systems, AMCIS 2008. .

SILVA, M. B. D.; NUNES, I. D.; NASCIMENTO, R. P. C; NUNES, M. A. S. N.

Iniciativas de Governança de TIC que sugerem Modelos, Técnicas e Boas Práticas para a Administração Pública Federal: um Mapeamento Sistemático

isys | Revista Brasileira de Sistemas de Informação, Rio de Janeiro, vol. 10, No. 1, pp. 39-51, 2017 
Becker, J., Bergener, P. and Räckers, M. (2009). Business process assessment and evaluation in public administrations using activity-based costing. In 15th Americas Conference on Information Systems 2009, AMCIS 2009. .

Bernardi, R. (2009). IT enactment of new public management in Africa: The case study of health information systems in Kenya. In Proceedings of the European Conference on e-Government, ECEG. .

Bigliardi, B. and Dormio, A. I. (2009). New management models of public administration: The italian experience of e-Government. In Proceedings of the European Conference on e-Government, ECEG. .

Carvalho, M. (2006) "Diretrizes para aplicação de Governança de TI nos órgãos públicos federais brasileiros usando o framework Cobit", Dissertação de Mestrado, UCB, Brasília, DF, Brasil.

. eGovernment development: The case of Macedonia. In Proceedings of the European Conference on e-Government, ECEG. .

Briganó, G. U., Brancher, J. D. and Barros, R. M. De (2012). Proposta de um Repositório de Conhecimentos em Governança de TIC. n. Sbsi, p. 654-659.

Cabiddu, F. (2010). The use of web services for inclusive decision process: Towards the enhancement of e-democracy. In Information Systems: People, Organizations, Institutions, and Technologies - ItAIS: The Italian Association for Information Systems. .

Capek, J. . and Ritschelova, I. . (2010). Secure communication between authorities, companies and citizens within eGovernment. In IMSCI 2010 - 4th International MultiConference on Society, Cybernetics and Informatics, Proceedings. .

Casagranda, M. ., Colazzo, L. . and Molinari, A. . (2014). Digital agenda and E-learning in Italian public administration. In Proceedings: DMS 2014 - 20th International Conference on Distributed Multimedia Systems. .

Casalino, N. . b and Draoli, M. . (2010). Governance and organizational aspects of an experimental groupware in the Italian public administration to support multiinstitutional partnerships. In Information Systems: People, Organizations, Institutions, and Technologies - ItAIS: The Italian Association for Information Systems. .

Castelnovo, W. (2009). Supporting innovation in small local government organizations. In Proceedings of the 3rd European Conference on Information Management and

Cernuzzi, L. . and Pane, J. . (2014). Toward open gGovernment in paraguay. IT Professional, v. 16, n. 5, p. 62-64.

Cerrillo-i-Martínez, A. (2012). A way to overcome the crisis in eGovernment: Cooperation among the regional and local authorities in Catalonia. In Proceedings of the European Conference on e-Government, ECEG. .

Consoli, S. ., Mongiovì, M. ., Nuzzolese, A. G. ., et al. (2015). A smart city data model based on semantics best practice and principles. In WWW 2015 Companion Proceedings of the 24th International Conference on World Wide Web. . 
Dandar, E., Sambuu, U., Unurkhaan, E. and Purev, T. (2007). Comprehensive analysis on Mongolian tax information system. In Proceedings of the 1st international conference on Theory and practice of electronic governance - ICEGOV '07. . ACM Press.

De Juana-Espinosa, S., Claver-Cortés, E. and Tarí, J. J. (2012). Barriers and facilitators to eGovernment in spanish municipalities: A study before and after the recession. In Proceedings of the European Conference on e-Government, ECEG.

Decman, M. and Klun, M. (2008). e-Tax services and their evolution: The case of Slovenia. In Proceedings of the European Conference on e-Government, ECEG. .

Dobnikar, A. and Nemec, A. Ž. (2007). eGovernment in Slovenia. Informatica (Ljubljana), v. 31, n. 4, p. 357-365.

Dwivedi, S. K. and Bharti, A. K. (2010). E-governance in India - problems and acceptability. Journal of Theoretical and Applied Information Technology, v. 17, n. 1, p. 37-43.

Emmanouil, C. I. . and Sotirios, V. . (2009). Business process reengineering as a modernizing tool for the public administration - From theory to reality. In 2009 4th Balkan Conference in Informatics, BCI 2009. .

Esteves, R. . and Alves, P. . (2013). Configuration management process implementation of ITIL framework: Case study - Culture, tourism and transport regional department of Madeira [Implementação do processo gestão da configuraç ão da framework ITIL: Estudo de caso - secretaria regional da cul. In Iberian Conference on Information Systems and Technologies, CISTI. .

Falivene, G. M. . and Silva, G. M. . b (2008). Reflections and proposals on public officials training and promotion of e-government. International Journal of Electronic Government Research, v. 4, n. 2, p. 43-58.

Galva, R. L. (2011). A tool for Monitoring the Public Administration in Dominican Republic: SISMAP. In ACM International Conference Proceeding Series. .

Garbarino, H. ., Delgado, B. . and Carrillo, J. . (2011). Taxonomy of it intangibles assets based on the electronic government maturity model in Uruguay. In Proceedings of the IADIS International Conference e-Commerce 2011, Part of the IADIS Multi Conference on Computer Science and Information Systems 2011, MCCSIS 2011. .

Gonzalez, R., Gasco, J. and Llopis, J. (2007). E-government success: Some principles from a Spanish case study. Industrial Management and Data Systems, v. 107, n. 6, p. 845-861.

Jackson, S. J. and Chongthammakun, R. (2011). Infrastructure and standards in Thai digital government. In ACM International Conference Proceeding Series. .

Juell-Skielse, G. and Perjons, E. (2009). Improving E-government through benefit analysis and value modeling. In Proceedings - International Computer Software and Applications Conference. .

Information Technology Governance Institute - ITGI (2016). "Cobit 5". Disponível em <www.isaca.org>. Acesso em Jun. de 2016

SILVA, M. B. D.; NUNES, I. D.; NASCIMENTO, R. P. C; NUNES, M. A. S. N.

Iniciativas de Governança de TIC que sugerem Modelos, Técnicas e Boas Práticas para a Administração Pública Federal: um Mapeamento Sistemático

isys | Revista Brasileira de Sistemas de Informação, Rio de Janeiro, vol. 10, No. 1, pp. 39-51, 2017 
Kitchenham, B., (2004). Procedures for performing systematic reviews. Keele, UK, Keele University, 33.

Ko, A., Kovács, B. and Gábor, A. (2011). Agile knowledge-based e-government supported by sake system. Journal of Cases on Information Technology, v. 13, n. 3, p. $1-20$.

Laszlo, G. and Szuts, I. (2006). e-Government development in Hungary. In ICEG 2006 2nd International Conference on e-Government. .

Liimatainen, K. and Seppänen, V. (2008). From fragmented e-Government projects towards national enterprise architecture programs. In Proceedings of the European Conference on e-Government, ECEG. .

Lin, Y. and Fong, S. (2013). Performance evaluation management model to accelerate the development of e-government in China. In ACM International Conference Proceeding Series. .

Lips, M. (2012). E-Government is dead: Long live public administration 2.0. Information Polity, v. 17, n. 3-4, p. 239-250.

Mătuescu, C. and Glăvan, O. R. (2012). Opportunities and limits of the application of eGovernment solutions at local level in Romania. In Proceedings of the European Conference on e-Government, ECEG. .

Mauro, A. and Augusto, A. (2010). Governança de TI em Instituições Federais de Ensino Superior.

Mohmand, A. M., Marjan, A. and Sangin, A. (2010). Developing e-government in Afghanistan. In Proceedings of the 4th International Conference on Theory and Practice of Electronic Governance - ICEGOV'10. . ACM Press.

Nguyen, H. T. T. (2008). Cultural barriers in preventing e-Government implementation in Asia: Evidence from Japan and Vietnam. In Proceedings of 4th International Conference on e-Government, ICEG 2008.

Niehaves, B., Müller-Wienbergen, F. and Becker, J. (2007). Public sector business intelligence -an open source approach. In Association for Information Systems - 13th Americas Conference on Information Systems, AMCIS 2007: Reaching New Heights.

Pankowska, M. (2011). Application of project portfolio management. In Information Systems Development: Asian Experiences. .

Pardhasaradhi, Y. . and Ahmed, S. . (2007). Efficiency of electronic public service delivery in India: Public-private partnership as a critical factor. In ACM International Conference Proceeding Series. .

Patra, M. R. . and Das, R. K. . (2014). Accessibility of e-Governance in rural India: A critical view point. In ACM International Conference Proceeding Series. .

Petersen, K., Feldt, R., Muftaba, S.; Mattson, M., (2008). Systematic mapping studies in software engineering. In: Proceedings of the 12th International Conference on Evaluation and Assessment in Software Engineering, 68-77. 
Rakotonirina, V. . and Raoelson, H. . (2013). E-government and performing enterprises Case of Madagascar. In ACM International Conference Proceeding Series. .

Reggi, L., Arduini, D., Biagetti, M. and Zanfei, A. (jun 2014). How advanced are Italian regions in terms of public e-services? The construction of a composite indicator to analyze patterns of innovation diffusion in the public sector. Telecommunications Policy, v. 38, n. 5-6, p. 514-529.

Rodríguez, J. F. D. (2013). Collective and participatory strategic planning of the department of Tolima in Colombia. In Proceedings of the 7th International Conference on Theory and Practice of Electronic Governance - ICEGOV'13.

Santos, P. ., Melo, A. I. . and Dias, G. P. . (2013). Administrative modernisation and egovernment: The case of Águeda [A modernização administrativa e o governo eletrónico: o caso de Águeda]. In Iberian Conference on Information Systems and Technologies, CISTI. .

Santos, V. . b and Varajão, J. . d (2015). PMO as a Key Ingredient of Public Sector Projects' Success - Position Paper. In Procedia Computer Science. .

Šimundić, S. and Boban, M. (2009). Development and infomatization of regional and local governments. In MIPRO 2009 - 32nd International Convention Proceedings: Digital Economy - 6th ALADIN, Information Systems Security, Business Intelligence Systems, Local Government and Student Papers. .

Stoll, M. and Laner, D. (2010a). System development by process integrated knowledge management. In Advanced Techniques in Computing Sciences and Software Engineering. .

Stoll, M. and Laner, D. (2010b). Service oriented e-government. In Innovations in Computing Sciences and Software Engineering. .

Suppa, A., Zardini, A., Rossignoli, C. and De Marco, M. (2013). ICT: The stone guest in the implementation of performance management system. The case of Italian Army. In Proceedings of the IADIS International Conference Information Systems 2013, IS 2013. . IADIS.

Tsohou, A. ., Lee, H. ., Irani, Z. ., et al. (2013). Proposing a reference process model for the citizen-centric evaluation of e-government services. Transforming Government: People, Process and Policy, v. 7, n. 2, p. 240-255.

Valtonen, K. and Leppänen, M. (2009). Business architecture development at public administration - Insights from government EA method engineering project in Finland. In Information Systems Development: Towards a Service Provision Society.

Verma, N. and Mishra, A. (2009). india.gov.in - India's approach in constructing onestop-solution towards e-government. In Proceedings of the 3rd International Conference on Theory and Practice of Electronic Governance - ICEGOV'09. 